\title{
Partial slip contact between a rigid punch with an arbitrary tip-shape and an elastic graded solid with a finite thickness
}

\author{
Peijian Chen, Shaohua Chen* \\ LNM, Institute of Mechanics, Chinese Academy of Sciences, Beijing 100190, China
}

\section{A R T I C L E I N F O}

\section{Article history:}

Received 19 September 2012

Available online 25 December 2012

\section{Keywords:}

Contact mechanics

Finite graded solid

Partial slip contact

Fretting

\begin{abstract}
A B S T R A C T
In contrast to the case of a graded half-space, a nonlinear plane strain partial slip model between a rigid punch with an arbitrary tip-shape and a finite graded solid is investigated. The modulus of the finite graded solid varies according to an exponential function in the thickness direction. Fourier integral transform method is adopted in order to reduce the current nonlinear problem to a set of singular integral equations. Based on the Goodman's approximation, the contact problem is simplified. An iterative method is used to determine the size of the contact zone, the interface stress singularity, as well as the distributions of the normal and tangential stresses. The effects of different parameters on the contact behavior, such as the ratio of shear modulus, the friction coefficient and the thickness of the graded solid, are analyzed. The results exhibit significant differences between the model of a half-space and the present one of a finite solid, which highlights the practicability of the present model for practical engineering applications and the design of novel graded materials.
\end{abstract}

(c) 2012 Elsevier Ltd. All rights reserved.

\section{Introduction}

Graded materials generally consist of several constituents with a gradual variation in the volume fracture from one location to the other (Guler and Erdogan, 2004). As a new kind of non-homogeneous composite, functionally graded materials (FGMs) possess special mechanical properties. As a coating or an interfacial material, it tends to reduce mismatching stresses, increase the bonding strength, improve surface properties, provide protection against adverse or chemical environment (Suresh and Mortensen, 1998) and possess excellent thermal shielding function, such as high temperature chambers, furnace liners, turbines, micro-electronics and space structures (Xing et al., 1998). Other potential applications of FGM involve contact with load transfer between or among different surfaces or interfaces of solids, generally with friction, such as cylinder linings, brake discs and other automotive components for

\footnotetext{
* Corresponding author. Tel.: +86 10 82543960; fax: +86 1082543977

E-mail address: chenshaohua72@hotmail.com (S. Chen).
}

the purpose of improving the wear resistance, as well as abradable seal design in stationary gas turbines (Guler and Erdogan, 2007).

Indentation techniques were used to measure the elastic properties of a graded material (Suresh et al., 1997; Jitcharoen et al., 1998; Krumova et al., 2001) and it was found that a power law or exponential one could be used to describe the variation tendency of Young's modulus of such a composite in the graded direction.

The Hertzian contact mechanics of graded materials have been studied by many researchers. Some representative work is given as follows. An axisymmetric contact problem of a graded half-space subjected to a concentrated load or flat, spherical and conical indenters was considered by Giannakopoulos and Suresh (1997a,b). It was found that the interface stress distribution under a frictionless indenter could be significantly influenced by the gradient features, even leading to a suppressive Hertzian crack near the contact edge. A closed-form analytical solution was further given by Giannakopoulos and Pallot (2000) for a two-dimensional contact model between a rigid cylinder 
and an elastic graded half-space. Guler and Erdogan (2004, $2006,2007)$ investigated the frictional contact model between a rigid punch and a FGM coated substrate as well as the one between a rigid punch and two layered-elastic solids with graded coatings. Contact model of a rigid punch on a homogeneous half-space with a graded coating was discussed by Chen and Chen (2012), in which the effects of the variation law and the thickness of the graded coating on the interfacial contact behaviors were discussed in detail. Receding contact between a graded coating and a homogeneous substrate was considered by El-Borgi et al. (2006) and Rhimi et al. (2009). Considering the thermo effects, Choi and Paulino $(2008,2010)$ analyzed the thermoelastic contact model for a coating/substrate system with or without a crack. Liu et al. (2011) investigated a plane strain frictional sliding contact model of a half-plane coated with graded materials. All the researches demonstrated that improving resistance of contact damage could be achieved by tuning the gradient index of the solid, the sliding speed of the punch and the friction coefficient of the contact interface.

As for the contact problem of a graded material considering the interface adhesion, typical works should be mentioned as follows. A plane strain adhesive contact model of a rigid cylinder on an elastic graded half-space was studied by Giannakopoulos and Pallot (2000), where the elastic modulus of the half-space varies with the depth according to a power law. Chen et al. (2009a,b) further discussed the plane strain model as well as an axis-symmetric one, in which a very simple closed-form analytical solution for the pull-off force was given. The corresponding non-slipping models were further discussed by Jin and Guo (2010) and Guo et al. (2011).

Fretting contact is often found in practical engineering, which involves cyclic contact stresses and may result in surface damage named as fretting (Elloumi et al., 2010). It has been extensively discussed for homogeneous materials (Ciavarella, 1998; Ciavarella and Hills, 1999; Hanson and Keer, 1989; Nowell et al., 1988; Spence, 1973; Wang et al., 2010). However, very limited literatures consider the fretting contact of FGM. Ke and Wang (2010) investigated a two-dimensional contact model of a half-space coated with a graded layer, which was subjected to a monotonically increasing normal load or a small-scale oscillatory tangential motion. A fully coupled partial slip contact problem of a graded half-plane was studied by Elloumi et al. (2010). In almost all of the existing fretting contact models concerning FGMs, an assumption of an infinite half-space was adopted, which is actually not consistent with the practical engineering. Is there any difference between the model of an infinite half-space and the one of a finite solid? How large is it if there is? When does the model of an infinite half-space can represent the one of a really finite solid? It should be very interesting and useful to answer these questions. In fact, similar questions have been put forward in lots of experiments and theoretical models concerning homogeneous layers (Araújo and Nowell, 1999; Argatov, 2001; Dini and Nowell, 2004; Fellows et al., 1995; Nowell and Hills, 1988). Especially, Fellows et al. (1995) pointed that "The geometry is relevant to a number of common fretting fatigue experiments" and "The half-plane solution may not give a good approximation to the stress component for practical experimental configurations, even where the specimen thickness is as large as 10 times the contact width".

In the present paper, a partial slip contact model of a rigid punch with an arbitrary end-tip and a graded solid with a finite thickness is investigated. One of the main objectives is to compare the fretting features of a homogeneous solid and a graded solid, the other one is to answer the above mentioned questions whether there are some differences between the model of a graded half-space and the one of a finite graded solid. The case with a normal load alone is investigated and a mixed boundary value problem is solved analytically using the singular equation method. An iterative procedure is adopted to find the size of the stick zone and the interface contact stresses. As special examples, cases of a flat punch and a cylindrical one are discussed in details with the help of numerical calculations.

\section{Partial slip contact model}

The partial slip contact model of a rigid punch with an arbitrary end-shape and a finite graded solid with a thickness $h$ is shown in Fig. 1, where a normal load $P$ is acted along the direction of $y$ axis to compress the rigid punch.

Due to the dissimilarity of the two contact bodies, the tangential displacements at the upper and lower contact interfaces are different so that interfacial slip or partial slip will generally take place (Johnson, 1985). In the contact model, we assume that the contact region lies in $-b \leqslant x \leqslant b$, which consists of an inner stick zone $|x| \leqslant a$ and two separated slip zones $a \leqslant|x| \leqslant b$, as shown in Fig. 1. In the slip zone, the tangential stress is assumed to be proportional to the normal contact pressure according to the Coulomb's friction law.

We assume that the Poisson's ratio of the finite graded solid is a constant $v$ and its shear modulus varies exponentially in the thickness direction as

$$
\begin{aligned}
& \mu_{2}(y)=\mu_{1} \exp (\gamma y), \quad 0 \leqslant y \leqslant h \\
& \gamma=\frac{1}{h} \ln \frac{\mu_{3}}{\mu_{1}},
\end{aligned}
$$

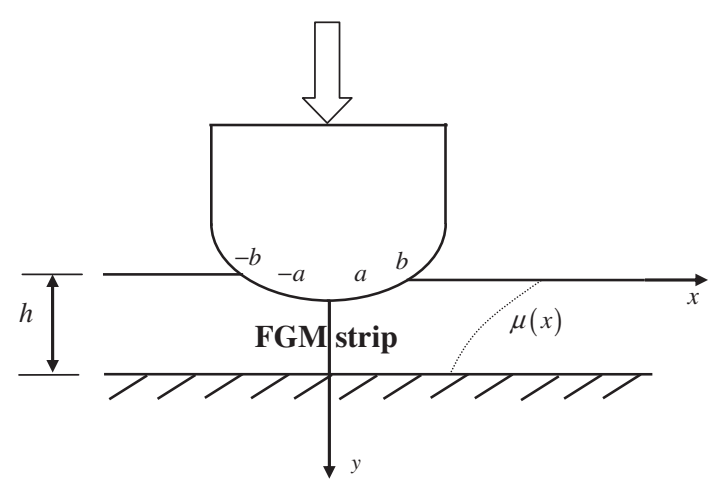

Fig. 1. Schematic of a partial slip contact model involving a finite graded layer fixed on a rigid foundation and a rigid punch. 
where the subscript " 1 " denotes the surface of the finite graded solid at $y=0$, and " 3 " is the lower boundary at $y=h$.

\section{Formulation of the contact model}

In the contact model, $u(x, y)$ and $v(x, y)$ denote the displacement components in the $x$ and $y$ directions, respectively. $\sigma_{x x}, \sigma_{y y}$ and $\sigma_{x y}$ are the stress components and the corresponding strain components are $\varepsilon_{x x}, \varepsilon_{y y}$ and $\varepsilon_{x y}$, respectively.

Neglecting the body force leads to the following equilibrium equations,

$$
\left\{\begin{array}{l}
\frac{\partial \sigma_{x x}}{\partial x}+\frac{\partial \sigma_{x y}}{\partial y}=0 \\
\frac{\partial \sigma_{x y}}{\partial x}+\frac{\partial \sigma_{y y}}{\partial y}=0
\end{array}\right.
$$

Due to an elastic problem considered in the present paper, the strain-displacement relationship and the constitutive relation can be expressed as

$\varepsilon_{x x}=\frac{\partial u}{\partial x}, \varepsilon_{x y}=\frac{1}{2}\left(\frac{\partial u}{\partial y}+\frac{\partial v}{\partial x}\right), \varepsilon_{y y}=\frac{\partial v}{\partial y}$,

and

$\left\{\begin{array}{l}\sigma_{x x}=\frac{\mu_{2}(y)}{\kappa-1}\left[(1+\kappa) \varepsilon_{x x}+(3-\kappa) \varepsilon_{y y}\right] \\ \sigma_{y y}=\frac{\mu_{2}(y)}{\kappa-1}\left[(1+\kappa) \varepsilon_{y y}+(3-\kappa) \varepsilon_{x x}\right] \\ \sigma_{x y}=2 \mu_{2}(y) \varepsilon_{x y}\end{array}\right.$

where $\kappa=3-4 v$ for a plane strain problem and $\kappa=(3-v) /(1+v)$ for a plane stress one.

Substituting Eqs. (4) and (5) into Eq. (3) yields partially differential equations in terms of the displacements as

$$
\left\{\begin{array}{c}
(\kappa-1) \frac{\partial^{2} u}{\partial y^{2}}+(\kappa+1) \frac{\partial^{2} u}{\partial x^{2}}+2 \frac{\partial^{2} v}{\partial \partial \partial y} \\
+\gamma(\kappa-1)\left(\frac{\partial v}{\partial x}+\frac{\partial u}{\partial y}\right)=0 \\
(\kappa-1) \frac{\partial^{2} v}{\partial x^{2}}+(\kappa+1) \frac{\partial^{2} v}{\partial y^{2}}+2 \frac{\partial^{2} u}{\partial x \partial y} \\
+\gamma\left[(\kappa+1) \frac{\partial v}{\partial y}+(3-\kappa) \frac{\partial u}{\partial x}\right]=0
\end{array}\right.
$$

and the following boundary conditions should be satisfied in the present model

$\sigma_{y y}(x, 0)=0, \sigma_{x y}(x, 0)=0, \quad|x|>b$,

$\sigma_{x y}(x, 0)=\eta \sigma_{y y}(x, 0) \operatorname{sign}(x), \quad a<|x|<b$,

$\sigma_{x y}(x, 0) \leqslant \eta\left|\sigma_{y y}(x, 0)\right|, \quad|x|<a$,

$u(x, h)=v(x, h)=0$.

where $\eta$ is the friction coefficient of the interface between the punch and the graded solid. The symbol sign $(\cdot)$ is a sign function. The main difference between a half-space model and a finite one is embodied by the boundary conditions in Eq. (10). If the thickness of the finite graded solid tends to be infinity, the present model will reduce to the half-space case (Elloumi et al., 2010).

According to the contact mechanics (Johnson, 1985), the interface normal displacement $v(x, 0)$ can be obtained from the profile of the above rigid punch, whose derivative is denoted as $\frac{\partial v(x, 0)}{\partial x}=f^{*}(x), \quad-b<x<b$.

In the stick zone, the relative tangential displacement on the interface is unknown for a non-conforming contact problem (Johnson, 1985) and its derivative is denoted as

$\frac{\partial u(x, 0)}{\partial x}=g^{*}(x), \quad-a<x<a$.

The relations between the interface stresses and the external load can be expressed as

$\int_{-b}^{b} \sigma_{y y}(x, 0) \mathrm{d} x=-P$

$\int_{-b}^{b} \sigma_{x y}(x, 0) \mathrm{d} x=0$

In the present paper, we define the unknown interface stresses as

$p(x)=-\sigma_{y y}(x, 0), \quad q(x)=-\sigma_{x y}(x, 0)$

In the slip zone, the shear stress is opposite to the slip direction, which is proportional to the interface normal pressure according to the Coulomb's friction law,

$q(x)= \begin{cases}-\eta p(x), & -b \leqslant x \leqslant-a, \\ \eta p(x), & a \leqslant x \leqslant b .\end{cases}$

Similar to Elloumi et al. (2010), we define the shear stress in the stick zone as the following form,

$q(x)=\eta p(x) \frac{x}{a}+q^{*}(x), \quad-a<x<a$,

where $q^{*}(x)$ is an unknown odd function and vanishes at $x= \pm a$.

Using the Fourier integral transform method with respect to Eq. (6) yields the displacement fields in the finite graded solid,

$\left\{\begin{array}{l}u(x, y)=\frac{1}{2 \pi} \int_{-\infty}^{+\infty} \sum_{j=1}^{4} B_{j}(s) e^{n_{j} y} e^{-i s x} \mathrm{~d} s \\ v(x, y)=-\frac{i}{2 \pi} \int_{-\infty}^{+\infty} \sum_{j=1}^{4} B_{j}(s) F_{j}(s) e^{\eta_{j} y} e^{-i s x} \mathrm{~d} s\end{array}\right.$

where $B_{j}(s)(j=1, \ldots, 4)$ are arbitrary unknown parameters, which will be determined by the boundary conditions. The terms $n_{j}(j=1, \ldots, 4)$ are roots of the following characteristic equation,

$\left(n^{2}+\gamma n-s^{2}\right)^{2}+\gamma^{2} s^{2}\left(\frac{3-\gamma}{1+\gamma}\right)=0$,

and can be obtained as,

$$
\begin{aligned}
n_{j} & =-\frac{\gamma}{2}+\sqrt{\frac{\gamma^{2}}{4}+s^{2}-i(-1)^{j} \gamma s\left(\frac{3-\kappa}{1+\kappa}\right)^{1 / 2}} \operatorname{Re}\left(n_{j}\right)>0, j \\
& =1,2
\end{aligned}
$$

$$
\begin{aligned}
n_{j} & =-\frac{\gamma}{2}-\sqrt{\frac{\gamma^{2}}{4}+s^{2}+i(-1)^{j} \gamma s\left(\frac{3-\kappa}{1+\kappa}\right)^{1 / 2}} \operatorname{Re}\left(n_{j}\right)<0, j \\
& =3,4
\end{aligned}
$$

$F_{j}(s)(j=1, \ldots, 4)$ are functions of $n_{j}(s)$ as follows, 


$$
\begin{aligned}
F_{j}(s) & =\frac{(\kappa-1)\left(n_{j}^{2}+\gamma n_{j}\right)-(1+\kappa) s^{2}}{\left[2 n_{j}+(\kappa-1) \gamma\right] s}, \\
(j & =1, \ldots, 4) .
\end{aligned}
$$

Substituting Eq. (18) and Eq. (4) into Eq. (5) yields the stress field in the finite graded solid,

$$
\left\{\begin{array}{c}
\sigma_{y y}(x, y)=\frac{\mu_{2}(y)}{1-\kappa} \frac{i}{2 \pi} \int_{-\infty}^{+\infty} \sum_{j=1}^{4} B_{j}(s)\left[(1+\kappa) F_{j} n_{j}+s(3-\kappa)\right] \\
\times e^{n_{j} y-i s x} \mathrm{~d} s \\
\tau_{x y}(x, y)=\frac{\mu_{2}(y)}{2 \pi} \int_{-\infty}^{+\infty} \sum_{j=1}^{4} B_{j}(s)\left[n_{j}+s F_{j}\right] e^{n_{j} y-i s x} \mathrm{~d} s
\end{array}\right.
$$

\section{Relation between the interface stress and displacement}

The interface contact stresses can be determined from the condition that the surface displacements of the finite graded solid should coincide with the profile of the rigid punch for a complete contact.

From the general solutions in Eqs. (18) and (22) and the constitutive relations in Eq. (5), the displacement and stress fields of the graded solid can be written in a Fourier-transformed domain as

$\mathbf{f}(s, y)=\Theta(s, y) \mathbf{a}(s)$

where $\mathbf{f}(s, y)$ is a state vector containing physical variables that need to be determined for the given constituents, $\mathbf{a}(s)$ is a vector for the four unknowns in the general solutions,

$\mathbf{f}(s, y)=\left\{\bar{v}(s, y) / i, \bar{u}(s, y), \bar{\sigma}_{y y}(s, y) / i, \bar{\tau}_{x y}(s, y)\right\}^{\mathrm{T}}$

$\mathbf{a}(s)=\left\{B_{1}(s), B_{2}(s), B_{3}(s), B_{4}(s)\right\}^{\mathrm{T}}$

$\Theta(s, y)$ is a $4 \times 4$ matrix, which is a function of not only the variables $s$ and $y$, but also the elastic parameters of the constituents.

On the bottom surface of the graded solid $y=h$, we have

$\mathbf{f}(s, h)=\Theta(s, h) \mathbf{a}(s)$

where

$\mathbf{f}(s, h)=\left\{0,0, \bar{\sigma}_{y y}(s, h) / i, \bar{\tau}_{x y}(s, h)\right\}^{\mathrm{T}}$

On the upper surface of the graded solid $y=0$, we have

$\mathbf{f}(s, 0)=\Theta(s, 0) \mathbf{a}(s)$

where

$\mathbf{f}(s, 0)=\left\{\bar{v}(s, 0) / i, \bar{u}(s, 0), \bar{\sigma}_{y y}(s, 0) / i, \bar{\tau}_{x y}(s, 0)\right\}$

Here, $\bar{\sigma}_{y y}(s, 0)$ and $\bar{\tau}_{x y}(s, 0)$ denote the transformed normal and tangential tractions on the upper surface of the graded solid. Therefore

$$
\begin{aligned}
\bar{\sigma}_{y y}(s, 0) & =\int_{-b}^{b} \sigma_{y y}(x, 0) e^{i s x} \mathrm{~d} x, \bar{\tau}_{x y}(s, 0) \\
& =\int_{-b}^{b} \tau_{x y}(x, 0) e^{i s x} \mathrm{~d} x
\end{aligned}
$$

Eliminating the unknown vector $\mathbf{a}(s)$ in Eqs. (26) and (28) yields

$\mathbf{f}(s, 0)=G(s) \mathbf{f}(s, h)$

where $\mathbf{G}(s)$ is a $4 \times 4$ transfer matrix connecting the upper and the lower surfaces of the graded solid,
$\mathbf{G}(s)=\Theta(s, 0) \Theta^{-1}(s, h)$

and the superscript " -1 " denotes the inverse of a matrix.

The corresponding displacements can be written as

$$
\begin{aligned}
v(x, 0)= & \frac{1}{2 \pi} \int_{-\infty}^{+\infty}\left[N_{11}(s) \bar{\sigma}_{y y}(s, 0)\right. \\
& \left.+i N_{12}(s) \bar{\tau}_{x y}(s, 0)\right] e^{-i s x} \mathrm{~d} s \\
u(x, 0)= & \frac{1}{2 \pi} \int_{-\infty}^{+\infty}\left[-i N_{21}(s) \bar{\sigma}_{y y}(s, 0)+N_{22}(s) \bar{\tau}_{x y}(s, 0)\right] e^{-i s x} \mathrm{~d} s \\
|x| \leqslant \infty &
\end{aligned}
$$

where the functions $N_{j k}(s)(j, k=1,2)$ are elements of a $2 \times 2$ matrix $N(s)$

$$
\mathbf{N}(s)=\left[\begin{array}{ll}
G_{13} & G_{14} \\
G_{23} & G_{24}
\end{array}\right]\left[\begin{array}{ll}
G_{33} & G_{34} \\
G_{43} & G_{44}
\end{array}\right]^{-1}
$$

Differentiating with respect to $x$, the integral Eqs. (33) and (34) can be rewritten as,

$$
\begin{aligned}
\frac{\partial v(x, 0)}{\partial x}= & -\frac{1}{2 \pi} \int_{-b}^{+b}\left[i K_{11}(x, r) \sigma_{y y}(r, 0)\right. \\
& \left.-K_{12}(x, r) \tau_{x y}(r, 0)\right] \mathrm{d} r \\
\frac{\partial u(x, 0)}{\partial x}= & -\frac{1}{2 \pi} \int_{-b}^{+b}\left[K_{21}(x, r) \sigma_{y y}(r, 0)\right. \\
& \left.+i K_{22}(x, r) \tau_{x y}(r, 0)\right] \mathrm{d} r
\end{aligned}
$$

where

$K_{j k}=\int_{-\infty}^{+\infty} s N_{j k}(s) e^{i s(r-x)} \mathrm{d} s, \quad j, k=1,2$

For a large value of $|s|$, we have the following asymptotic solutions,

$\lim _{|s| \rightarrow \infty} s N_{11}(s)=\lim _{|s| \rightarrow \infty} s N_{22}(s)=N_{1}^{\infty} \frac{s}{|s|}$,

$\lim _{|s| \rightarrow \infty} s N_{12}(s)=\lim _{|s| \rightarrow \infty} s N_{21}(s)=N_{2}^{\infty}$,

where $N_{1}^{\infty}=-\frac{\kappa+1}{4 \mu_{1}}$ and $N_{2}^{\infty}=-\frac{\kappa-1}{4 \mu_{1}}$.

Separating the leading term from the kernels in Eq. (38) and using Eqs. (11), (12), (15) as well as the following generalized functions

$$
\begin{aligned}
\int_{0}^{\infty} \sin [s(r-x)] \mathrm{d} s & =\frac{1}{r-x}, \quad \int_{0}^{\infty} \cos [s(r-x)] \mathrm{d} s \\
& =\pi \delta(r-x)
\end{aligned}
$$

Eqs. (36) and (37) can be written as,

$\frac{1}{\pi} \int_{-b}^{b} \frac{p(r)}{x-r} \mathrm{~d} r-\frac{\kappa-1}{\kappa+1} q(x) d+\frac{4 \mu_{1}}{\pi(\kappa+1)} \int_{-b}^{b} k_{11}(x, r) p(r) \mathrm{d} r$
$+\frac{4 \mu_{1}}{\pi(\kappa+1)} \int_{-b}^{b} k_{12}(x, r) q(r) \mathrm{d} r=-\frac{4 \mu_{1}}{(\kappa+1)} f^{*}(x)$,

$\frac{1}{\pi} \int_{-b}^{b} \frac{q(r)}{x-r} \mathrm{~d} r-\frac{\kappa-1}{\kappa+1} p(x)-\frac{4 \mu_{1}}{\pi(\kappa+1)} \int_{-b}^{b} k_{21}(x, r) p(r) \mathrm{d} r$

$+\frac{4 \mu_{1}}{\pi(\kappa+1)} \int_{-b}^{b} k_{22}(x, r) q(r) \mathrm{d} r=-\frac{4 \mu_{1}}{(\kappa+1)} g^{*}(x)$, 
where $\delta(\cdot)$ is the Dirac delta function and $k_{j k}(x, r)(j, k=1,2)$ is defined as

$$
\begin{aligned}
k_{j k}(x, r) & =\int_{0}^{+\infty}\left[s N_{j k}(s)-N_{1}^{\infty}\right] \sin s(r-x) \mathrm{d} s, \\
(j, k) & =(1,1),(2,2), \\
k_{j k}(x, r) & =\int_{0}^{+\infty}\left[s N_{j k}(s)-N_{2}^{\infty}\right] \cos s(r-x) \mathrm{d} s, \\
(j, k) & =(1,2),(2,1) .
\end{aligned}
$$

Here, one can see that the interface normal and tangential stresses are coupled in the Cauchy singular Eqs. (43) and (44). Combining Eqs. (11)-(17), a well-posed problem is established and the solution can be obtained by numerical techniques, which are given in the next section.

\section{Numerical solutions of the singular integral equations}

According to Elloumi et al. (2010), the coupling effect of normal and tangential stresses on the final results is not significant in contrast to the results in an uncoupled problem, when only a normally external load is acted on the above rigid punch. In the present paper, an approximation that is often called as the Goodman approximation is adopted to neglect the influence of tangential traction on the interface normal stress, whereas the influence of the normal stress on the tangential one still exists. Then, Eq. (41) can be simplified as

$\frac{1}{\pi} \int_{-b}^{b} \frac{p(r)}{r-x} \mathrm{~d} r+\frac{1}{\pi \alpha_{1}} \int_{-b}^{b} k_{11}(x, r) p(r) \mathrm{d} r=-\frac{1}{\alpha_{1}} f^{*}(x),|x|<b$

where $\alpha_{1}=-\frac{(\kappa+1)}{4 \mu_{1}}$.

Introducing the following normalized quantities,

$r=b \lambda, \quad x=b \zeta$,

Eqs. (45) and (13) can be rewritten as

$\frac{1}{\pi} \int_{-1}^{1} \frac{p(\lambda)}{\lambda-\zeta} \mathrm{d} \lambda+\frac{b}{\pi \alpha_{1}} \int_{-1}^{1} k_{11}(\zeta, \lambda) p(\lambda) \mathrm{d} \lambda=-\frac{1}{\alpha_{1}} f^{*}(\zeta),|\zeta|<1$

$\int_{-1}^{1} p(\lambda) \mathrm{d} \lambda=\frac{P}{b}$

For a given value $b$ of the contact region or a given load $P$, the interface normal stress $p(x)$ can be obtained from Eqs. (47) and (48). Then, substituting Eq. (17) into (42) yields the equation about $q^{*}(x)$,

$\frac{1}{\pi} \int_{-a}^{a} \frac{q^{*}(r)}{x-r} \mathrm{~d} r-\frac{1}{\pi \alpha_{1}} \int_{-a}^{a} k_{22}(x, r) q^{*}(r) \mathrm{d} r=N(x),|x|<a$,

where

$$
\begin{aligned}
N(x)= & \frac{1}{\alpha_{1}} \frac{\partial u(x, 0)}{\partial x}-\frac{\kappa-1}{\kappa+1} p(x)-\frac{1}{\pi \alpha_{1}} \int_{-b}^{b} k_{21}(x, r) p(r) \mathrm{d} r \\
& +\frac{\eta}{\pi} \int_{-b}^{-a} \frac{p(r)}{x-r} \mathrm{~d} r-\frac{\eta}{\pi} \int_{a}^{b} \frac{p(r)}{x-r} \mathrm{~d} r-\frac{\eta p(a)}{\pi a} \int_{-a}^{a} \frac{r}{x-r} \mathrm{~d} r \\
& -\frac{1}{\pi \alpha_{1}}\left[\int_{-b}^{-a} \eta k_{22}(x, r) p(r) \mathrm{d} r-\frac{\eta p(a)}{a} \int_{-a}^{a} k_{22}(x, r) r \mathrm{~d} r\right. \\
& \left.-\int_{a}^{b} \eta k_{22}(x, r) p(r) \mathrm{d} r\right] .
\end{aligned}
$$

Introducing the following normalized quantities,

$r=a s, \quad x=a z$

Eq. (49), which is only effective in the stick zone $|x|<a$, may be reformulated as

$$
\begin{aligned}
& \frac{1}{\pi} \int_{-1}^{1} \frac{q^{*}(s)}{z-s} \mathrm{~d} s-\frac{a}{\pi \alpha_{1}} \int_{-1}^{1} k_{22}(z, s) q^{*}(s) \mathrm{d} s=N(z), \quad|z| \\
& \quad<1
\end{aligned}
$$

In the following sub-sections, special cases of a flat punch and a circular one will be analyzed in details.

\subsection{Flat punch case}

In the contact model of a flat punch, we have (Johnson, 1985)

$v(x, 0)=$ constant $, \quad \frac{\partial v(x, 0)}{\partial x}=0, \quad|x|<b$,

Then, according to Eq. (11), we have $f^{*}(x)=0$.

Due to a vanishing tangential displacement in the stick zone, the boundary condition in Eq. (12) becomes

$\frac{\partial u(x, 0)}{\partial x}=0, \quad|x|<a$.

In the contact model of a flat punch, the unknown normal stress $p(\lambda)$ is unbounded, which may be expressed as (Erdogan et al., 1973)

$p(\lambda)=\frac{f(\lambda)}{\sqrt{1-\lambda^{2}}}$

While $q^{*}(s)$ is bounded, which is usually denoted as (Erdogan et al., 1973)

$q^{*}(s)=\phi(s) \sqrt{1-s^{2}}$,

where $f(\lambda)$ and $\phi(s)$ are two continuous functions and bounded in the interval $[-1,1]$.

In addition, Erdogan et al. (1973) has verified that the solution of Eq. (52) should satisfy the following consistency condition (Muskhelishvili, 1953),

$\int_{-1}^{1}\left[\frac{1}{\pi} \int_{-1}^{1} \frac{q^{*}(s)}{z-s} \mathrm{~d} s\right] \frac{\mathrm{d} z}{\sqrt{1-z^{2}}}=0$.

Substituting Eq. (52) into the above consistency condition yields

$$
\begin{aligned}
& \int_{-1}^{1}\left[\frac{1}{\pi} \int_{-1}^{1}\left\{N(z)-\frac{a}{\pi \alpha_{1}} \int_{-1}^{1} k_{22}(z, s) q^{*}(s) \mathrm{d} s\right\} \mathrm{d} s\right] \frac{\mathrm{d} z}{\sqrt{1-z^{2}}} \\
& =0 .
\end{aligned}
$$

With the help of Eqs. (55) and (56) and the GaussChebyshev quadrature method (Erdogan et al., 1973), Eqs. (47), (48), and (52) can be reduced to a set of linear algebraic equations as follows,

$$
\begin{aligned}
& \frac{1}{M} \sum_{l=1}^{M} f\left(\lambda_{l}\right)\left[\frac{\alpha_{1}}{\lambda_{l}-\zeta_{m}}+b k_{11}\left(\zeta_{m}, \lambda_{l}\right)\right]=0, \quad(m \\
& \quad=1,2, \ldots, M-1)
\end{aligned}
$$


$\frac{1}{M} \sum_{l=1}^{M} f\left(\lambda_{l}\right)=\frac{P}{\pi b}$

$$
\begin{aligned}
& \sum_{l=1}^{M} \frac{\left(1-s_{l}^{2}\right) \phi\left(s_{l}\right)}{M+1}\left[\frac{1}{z_{i}-s_{l}}-\frac{a}{\alpha_{1}} k_{22}\left(z_{i}, s_{l}\right)\right]=N\left(z_{i}\right) \\
& \quad(i=1,2, \ldots, M+1)
\end{aligned}
$$

where

$$
\begin{aligned}
& \lambda_{l}=\cos \frac{(2 l-1) \pi}{2 M}, \quad l=1,2, \ldots, M, \\
& \zeta_{m}=\cos \frac{\pi m}{M}, \quad m=1,2, \ldots, M-1, \\
& s_{l}=\cos \frac{l \pi}{M+1}, \quad l=1,2, \ldots, M, \\
& z_{i}=\cos \frac{\pi(2 i-1)}{2(M+1)}, \quad i=1,2, \ldots, M+1 .
\end{aligned}
$$

There are $2 M+1$ unknowns in Eqs. (58) and (59), i.e., $a$, $f\left(\lambda_{l}\right)$ and $\phi\left(s_{l}\right)(l=1,2, \ldots, M)$, which can be found using the following iteration procedure,

(1) $f\left(\lambda_{l}\right)(l=1,2, \ldots, M)$ can be obtained directly from the linear algebraic Eqs. (59a) and (59b). Then, the interface normal stress $p(\lambda)$ can be found from Eq. (55).

(2) According to the approximate expression for the case of a homogeneous half-plane (Spence, 1973), we choose an initial value for $a$.

(3) $\phi\left(s_{l}\right)(l=1,2, \ldots, M)$ can be achieved from Eq. (59c), in which the $(M / 2+1)$ th equation is satisfied automatically.

(4) Substituting the achieved $\phi\left(s_{l}\right)(l=1,2, \ldots, M)$ into Eq. (58) yields an updated $a$. Then, using step (3) to find updated $\phi\left(s_{l}\right)(l=1,2, \ldots, M)$. Iterations go like this until the difference of results between two neighboring steps is sufficiently small, then, the results are recorded.

(5) Once $\phi\left(s_{l}\right)(l=1,2, \ldots, M)$ are obtained, the normalized tangential stress in Eq. (56) is found.

The stress intensity factors at points $x= \pm b$ can be obtained from the interface normal stress $p(\lambda)$,

$$
\begin{aligned}
& F_{I}(b)=\lim _{r \rightarrow b} \frac{p(r)}{2^{-1 / 2}(b-r)^{-1 / 2}}=f(1) b^{1 / 2}, \\
& F_{I}(-b)=\lim _{r \rightarrow-b} \frac{p(r)}{2^{-1 / 2}(b+r)^{-1 / 2}}=f(-1) b^{1 / 2} .
\end{aligned}
$$

\subsection{Plane strain case with a cylindrical punch}

According to Johnson (1985), the normal displacement at the contact interface and its derivative in the plane strain contact problem with a cylindrical punch can be written as, $v(x, 0)=\frac{x^{2}}{2 R}, \quad \frac{\partial v(x, 0)}{\partial x}=\frac{x}{R}, \quad|x|<b, \quad f^{*}(x)=\frac{x}{R}$

According to Spence (1968), we assume the points in the central stick zone are pre-strained before sticking and the pre-strain is proportional to $|x|$. Then the boundary condition given in Eq. (12) can be written as

$\frac{\partial u(x, 0)}{\partial x}=C|x|, \quad|x|<a$

where $C$ is an unknown constant.

The interface normal stress $p(\lambda)$ in the plane strain case with a cylindrical punch should be bounded, which may be expressed as (Erdogan et al., 1973)

$p(\lambda)=f(\lambda) \sqrt{1-\lambda^{2}}$.

$q^{*}(s)$ in Eq. (52) is also a bounded function, which is usually given as the following form (Erdogan et al., 1973)

$q^{*}(s)=\phi(s) \sqrt{1-s^{2}}$.

Functions $f(\lambda)$ and $\phi(s)$ in Eqs. (64) and (65) are continuous and bounded in the interval $[-1,1]$. The consistency condition in Eq. (58) should also be satisfied in such a cylindrical punch case.

With the help of Eqs. (64) and (65) and the GaussChebyshev quadrature method (Erdogan et al., 1973), integral Eqs. (47), (48), and (52) can be reduced as

$\sum_{l=1}^{M} \frac{\left(1-\lambda_{l}^{2}\right)}{M+1} f\left(\lambda_{l}\right)\left[\frac{1}{\lambda_{l}-\zeta_{m}}+\frac{b}{\alpha_{1}} k_{11}\left(\zeta_{m}, \lambda_{l}\right)\right]$
$\quad=\frac{b \zeta_{m}}{\alpha_{1} R},(m=1,2, \ldots, M+1)$

$\sum_{l=1}^{M} \frac{\left(1-\lambda_{l}^{2}\right)}{M+1} f\left(\lambda_{l}\right)=\frac{P}{\pi b}$

$\sum_{l=1}^{M} \frac{\left(1-s_{l}^{2}\right) \phi\left(s_{l}\right)}{M+1}\left[\frac{1}{z_{i}-s_{l}}-\frac{a}{\alpha_{1}} k_{22}\left(z_{i}, s_{l}\right)\right]-\frac{1}{\alpha_{1}} C\left|a z_{i}\right|$

$=N\left(z_{i}\right),(i=1,2, \ldots, M+1)$

where

$\lambda_{l}=\cos \frac{l \pi}{M+1}, \quad l=1,2, \ldots, M$,

$\zeta_{m}=\cos \frac{\pi(2 m-1)}{2(M+1)}, \quad m=1,2, \ldots, M+1$,

$s_{l}=\cos \frac{l \pi}{M+1}, \quad l=1,2, \ldots, M$,

$z_{i}=\cos \frac{\pi(2 i-1)}{2(M+1)}, \quad i=1,2, \ldots, M+1$.

Since the solution of Eq. (47) is bounded at the contact edges, it must satisfy the consistency condition. According to Erdogan et al. (1973), the $(M / 2+1)$ th equation in Eq. (66a) corresponds to the consistency condition, which is always satisfied.

Thus, Eqs. (58) and $(66 a-c)$ consist of $(2 M+3)$ independent equations including $(2 M+3)$ unknowns, i.e., $f\left(\lambda_{l}\right), \phi$ 
$\left(s_{l}\right), a, P($ or $b)$ and $C$, which can be found by the iteration method similar to that used in the flat punch case.

\section{Results and discussions}

\subsection{Special cases}

In our model, if the graded parameter $\gamma\left(=\frac{1}{h} \log \frac{\mu_{3}}{\mu_{1}}\right)$ vanishes and the contact width is much smaller than the thickness of the graded solid, the present model will be reduced to a special one, i.e., the partial slip contact problem of a homogeneous half-space under plane strain condition (Spence, 1973; Nowell et al., 1988). Fig. 2 shows the distribution of the dimensionless contact stresses $p(y) / \sigma_{0}$ and $q(y) / \sigma_{0}$ in the contact model of a flat punch contacting a homogeneous half-space, where $\sigma_{0}$ is the average contact pressure. The size of the stick zone varying with the friction coefficient for a fixed Poisson's ratio is shown in Fig 3. One can readily see that the present results agree very well with the Spence's ones. As for the plane strain model of a rigid circular punch contacting a homogeneous half-plane, Fig. 4 shows the distributions of the contact stress obtained from the present reduced model and that
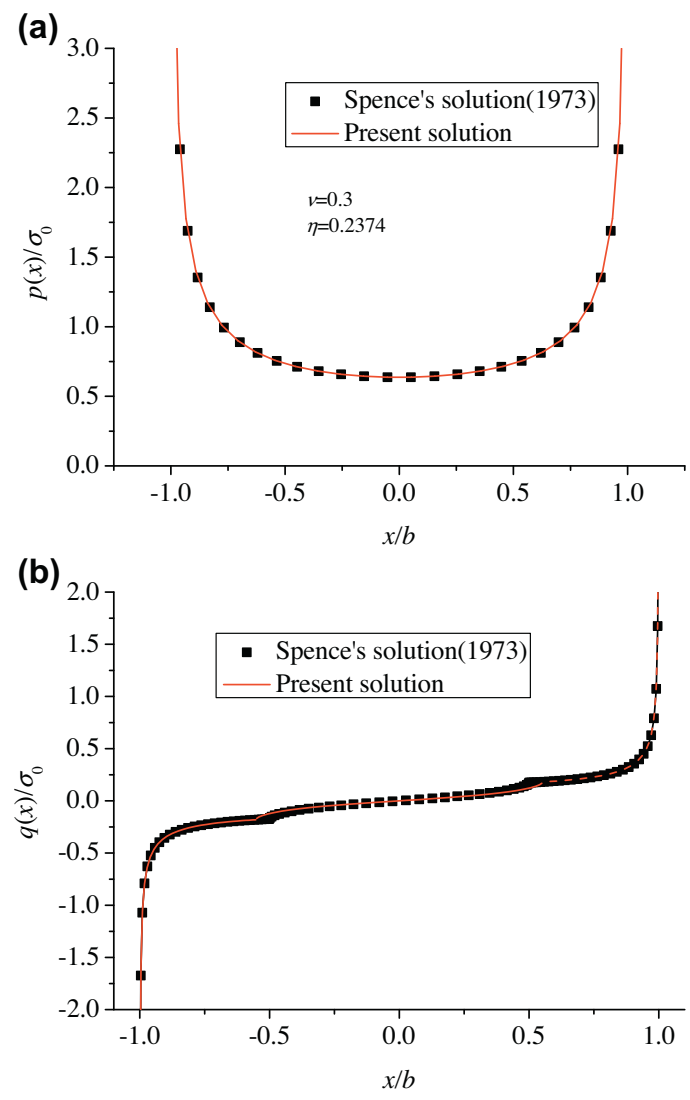

Fig. 2. Validation of normalized contact stress distribution of a homogeneous half-plane in the case of a flat punch (a) normal stress; (b) shear stress; $\eta=0.2374$ and $v=0.3$.

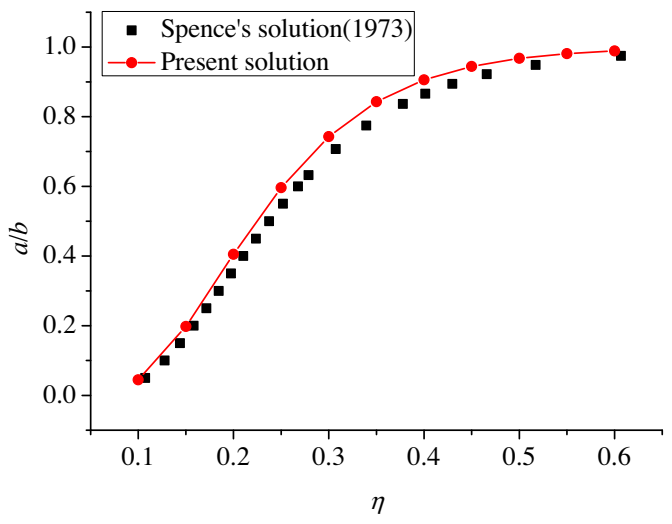

Fig. 3. Validation of the stick size $a / b$ versus the friction coefficient of a homogeneous half-plane in the case of a flat punch, $\eta ; v=0.3$.
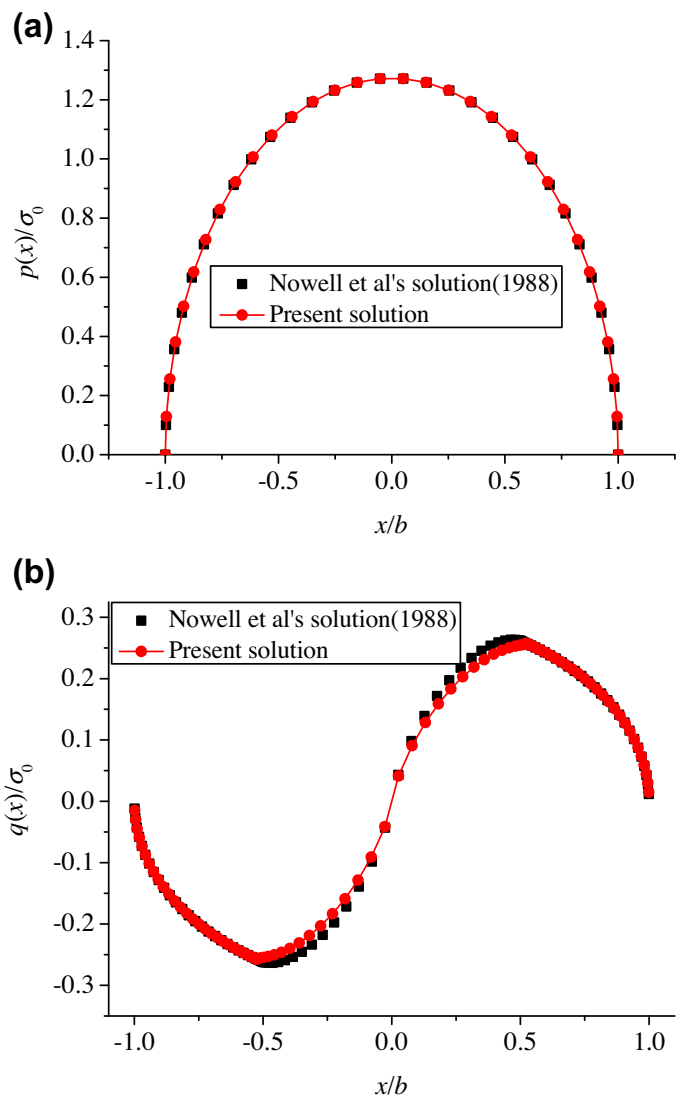

Fig. 4. Validation of normalized contact stress distribution of a homogeneous half-plane in the case of a circular punch (a) normal stress; (b) shear stress; $\eta=0.2374$ and $v=0.3$.

from Nowell et al. (1988) with parameters $\eta=0.23738$ and $v=0.3$. Consistent results are achieved for both the present model and the one in Nowell et al. (1988).

If the graded parameter $\gamma$ is chosen as a constant and the contact width is much smaller than the thickness of the graded solid, i.e., $b \ll h$, the present model can be 


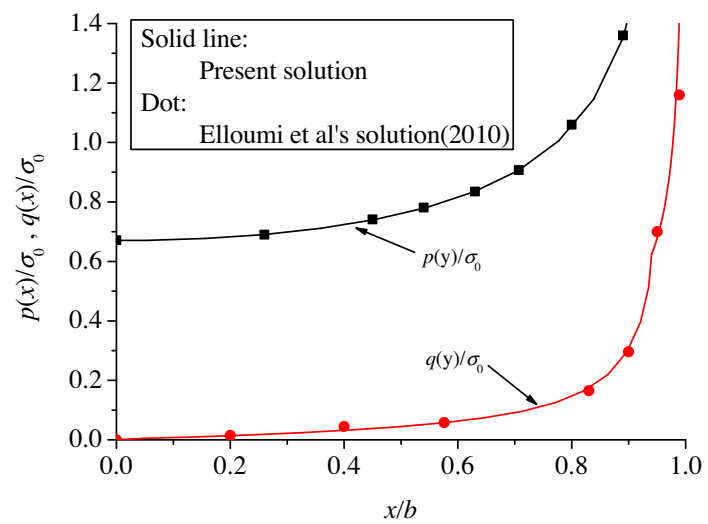

Fig. 5. Validation of normalized contact stress distribution of a graded half-plane with $\gamma b=0.1$ in the case of a flat punch; $\eta=0.35$ and $v=0.3$.
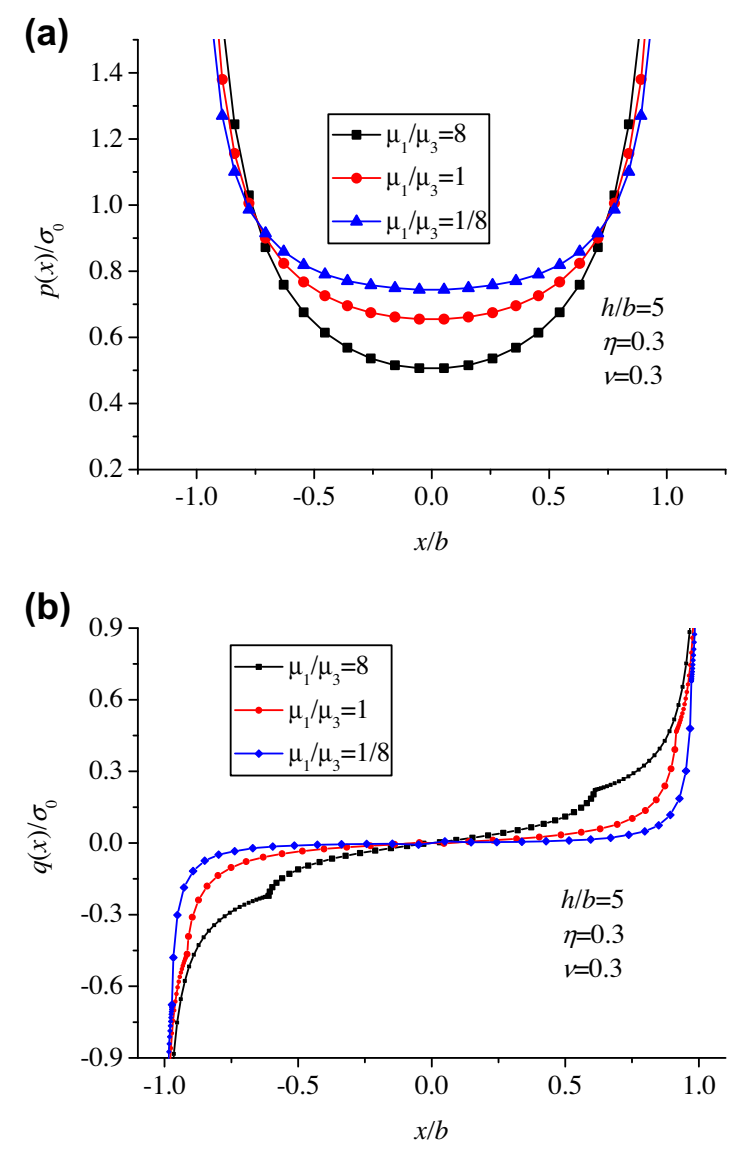

Fig. 6. Distributions of normalized contact stress distribution in the case of a flat punch for some selected ratio of shear moduli with $\eta=0.3, h / b=5$ and $v=0.3$ (a) normal stress; (b) shear stress.

reduced to another special one, i.e., the partial slip contact model between a rigid punch and a graded half-plane (Elloumi et al., 2010; Kallel-Kamoun et al., 2010). Numerical results for the present model and the graded half-plane model (Elloumi et al., 2010; Kallel-Kamoun et al., 2010) are
Table 1

Non-dimensional stress intensity factors $F_{l}(b) / F_{0}$ at the contact edge for different values of $h / b$ and $\mu_{1} / \mu_{3}$ with $\eta=0.3$ and $v=0.3$.

\begin{tabular}{llll}
\hline$\mu_{1} / \mu_{3}$ & $h / b=20$ & $h / b=10$ & $h / b=5$ \\
& $F_{l}(b) / F_{0}$ & $F_{l}(b) / F_{0}$ & $F_{l}(b) / F_{0}$ \\
\hline $1 / 8$ & 0.2930 & 0.2801 & 0.2536 \\
1 & 0.3178 & 0.3161 & 0.3098 \\
8 & 0.3450 & 0.3699 & 0.4075 \\
\hline
\end{tabular}

(a)

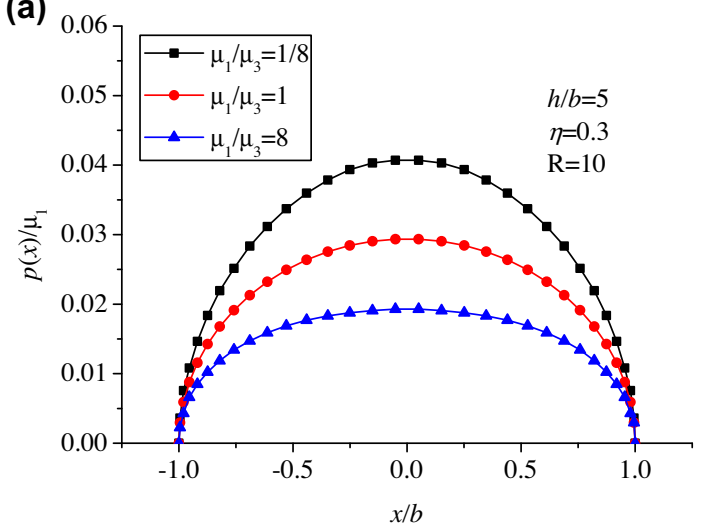

(b)

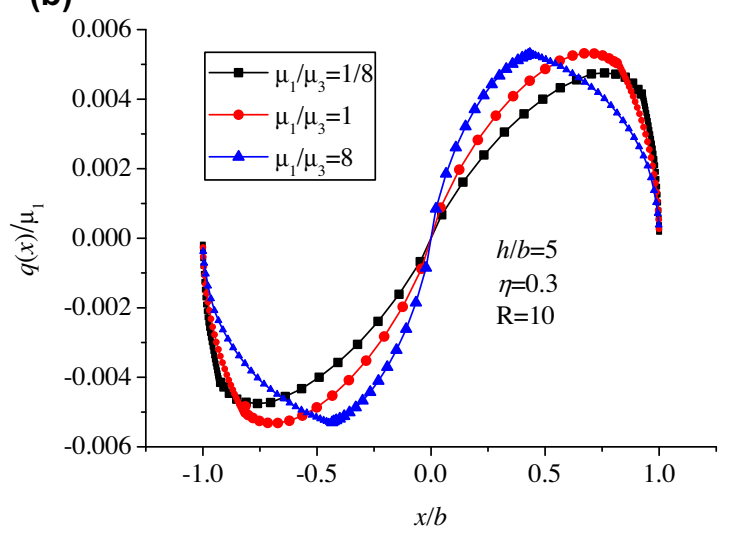

Fig. 7. Distributions of normalized contact stress distribution in the case of a circular punch for some selected ratio of shear moduli with $\eta=0.3, h$ / $b=5, R=10$ and $v=0.3$ (a) normal stress; (b) shear stress.

shown in Fig. 5, where it shows good agreement between the two kinds of models.

\subsection{Parametric study}

Fig. 6( $a$ and $b)$ illustrate the effect of shear modulus ratio $\mu_{1} / \mu_{3}$ on the distributions of the normal contact stress and the tangential one in the partial slip contact model of a flat punch and a finite graded solid, respectively. From Fig. 6(a), one can see that the non-dimensional normal contact stress $p(x) / \sigma_{0}$ becomes more uniform in the contact area with a decreasing $\mu_{1} / \mu_{3}$. The corresponding nondimensional stress intensity factors $F_{I}(b) / F_{0}$ are shown in Table 1 for different ratios of shear modulus $\mu_{1} / \mu_{3}$, where 
$F_{0}=\mathrm{Pb}^{-1 / 2}$. Table 1 exhibits that the stress intensity factor for the case of $\mu_{1} / \mu_{3}<1$ is smaller than that for the case of $\mu_{1} / \mu_{3}>1$. From the fretting mechanics point of view, it is easy to conclude that a graded layer with gradually increasing stiffness in the thickness direction will have less possibility for crack initiation and propagation in the flat punch case. Fig. 6(b) shows that, for a fixed $\eta$, a decreasing $\mu_{1} / \mu_{3}$ leads to an increase of the size of stick zone $a / b$.

Fig. 7 illustrates the effect of shear modulus ratio $\mu_{1} / \mu_{3}$ on the distributions of the normal and tangential contact stresses for the case of a circular punch. For fixed $\eta$ and $b / h$, it is easy to find that a smaller ratio of shear modulus $\mu_{1} / \mu_{3}$ results in a larger magnitude of contact pressure in Fig. 7(a). It also means that for the same normal loading, a smaller $\mu_{1} / \mu_{3}$ corresponds to a narrower contact width. Both Bramhall (1973) and Nowell (1988) have found that a large contact width results in a short fretting fatigue life. Thus, a graded layer with gradually increasing stiffness in the thickness direction, i.e., $\mu_{1} / \mu_{3}<1$, should have a long fretting fatigue life. The distribution of the tangential contact stress is shown in Fig 7(b), where one can see that for a fixed $\eta$, a decreasing $\mu_{1} / \mu_{3}$ leads to an increase size of the stick zone $a / b$.

Fig. 8( $a$ and b) exhibit the effect of the friction coefficient $\eta$ on the size of the stick zone $a / b$ for cases of a flat
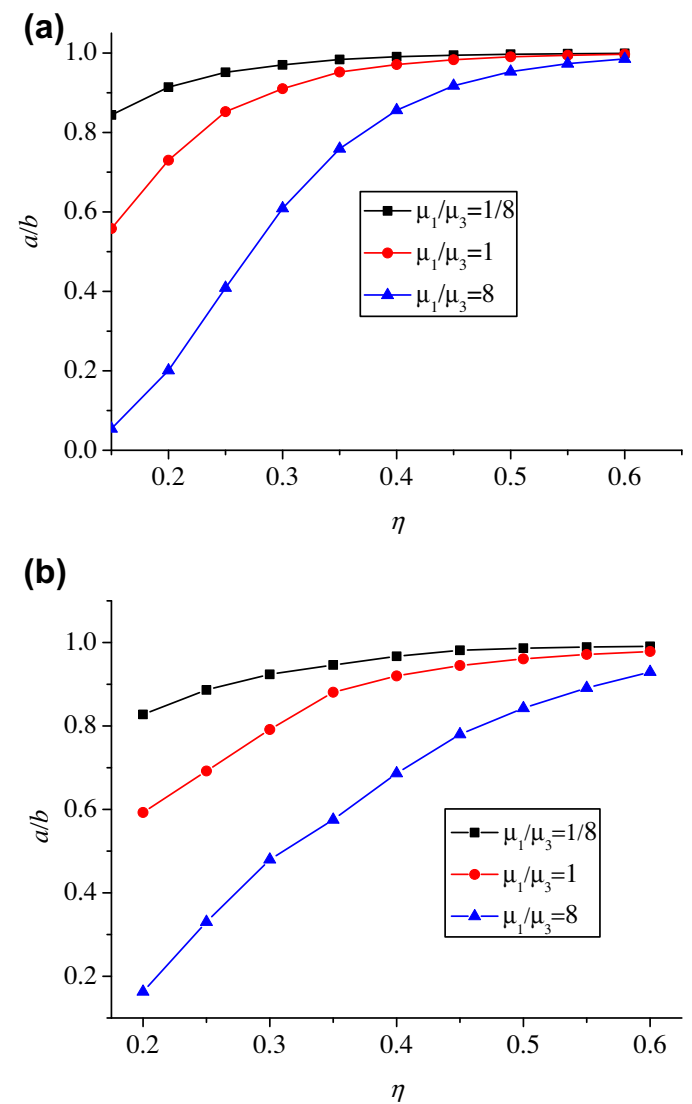

Fig. 8. Variations of the stick zone versus the friction coefficient, $\eta$ for some selected ratio of shear moduli with $h / b=5$ and $v=0.3$ (a) flat punch; (b) circular punch $(R=10)$.

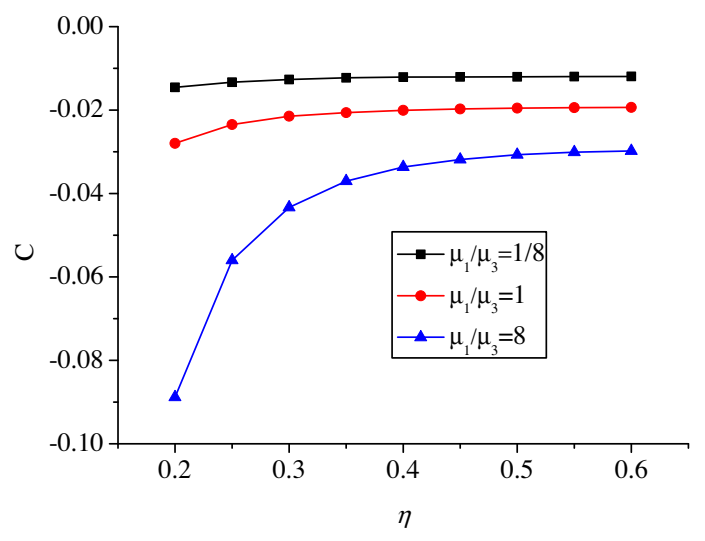

Fig. 9. Variations of the slope of the tangential displacement gradient versus the friction coefficient, $\eta$ for the case of circular punch and some selected ratio of shear moduli with $h / b=5, v=0.3$ and $R=10$.
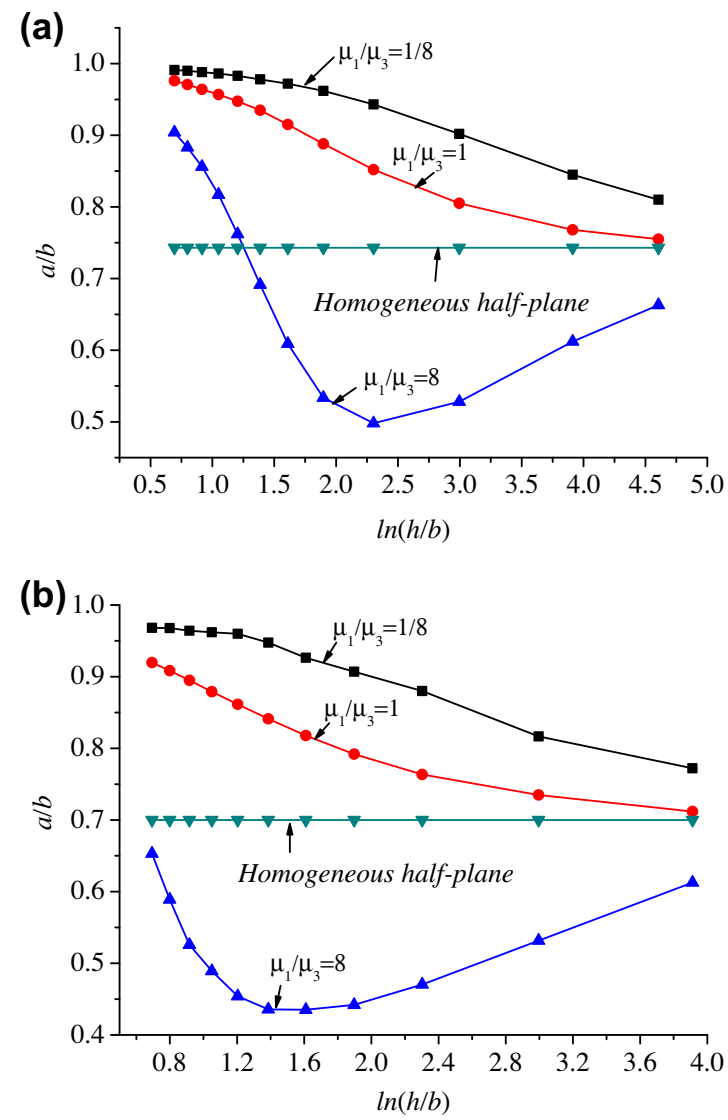

Fig. 10. Variations of the stick zone versus the thickness of the graded layer, $\ln (h / b)$ for some selected ratio of shear moduli with $\eta=0.3$ and $v=0.3$, compared with the homogeneous half-plane case (a) flat punch; (b) circular punch $(R=10)$.

punch and a circular one, respectively, where the ratio of shear modulus $\mu_{1} / \mu_{3}$ is fixed. It is easy to find the fact that an increasing friction coefficient will lead to an increasing stick zone and finally it will tend to be a fully adhesive 
contact. It means a larger friction coefficient may relieve the occurrence of interfacial slip. For a fixed $\eta$, decreasing $\mu_{1} / \mu_{3}$ will also result in an increasing stick zone.

The slope of the tangential displacement $C$ in Eq. (63) is an additional unknown for the case of a circular punch. Fig. 9 shows the effect of the shear modulus ratio $\mu_{1} / \mu_{3}$ on the relation between $C$ and the friction coefficient $\eta$.
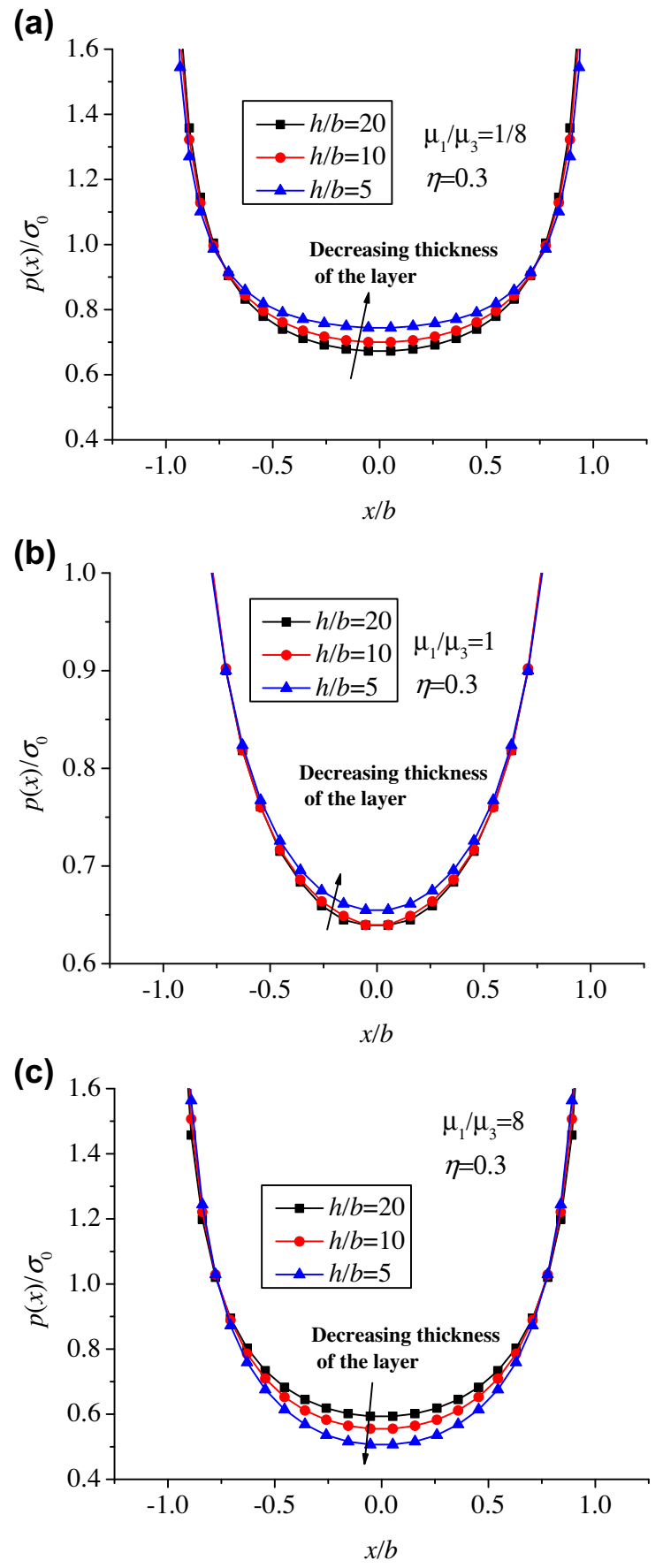

Fig. 11. Distributions of normalized contact stress distribution in the case of a flat punch for some selected thickness of the graded layer with $\eta=0.3$ and $v=0.3$ (a) normal stress $\left(\mu_{1} / \mu_{3}=1 / 8\right)$; (b) normal stress $\left(\mu_{1} / \mu_{3}=1\right)$; (c) normal stress $\left(\mu_{1} / \mu_{3}=8\right)$.
One can see that with the increase of $\eta$, the value of $C$ tends to be a constant. For a determined $\eta$, the absolute value of $C$ will increase with an increasing $\mu_{1} / \mu_{3}$.

The relation between the thickness of the graded layer $h / b$ and the stick zone $a / b$ with different $\mu_{1} / \mu_{3}$ for cases of a flat punch and a circular one are given in Fig. 10(a and $b$ ), respectively. With the increase of the non-dimensional thickness of the graded layer $h / b$, all the results will tend to that of a homogeneous half-space case due to the weakened boundary effect and slow variation of shear modulus in the thickness direction. It is interesting to find that the result for a homogeneous finite layer case, i.e., $\mu_{1} /$ $\mu_{3}=1$, deviates significantly from that for the homogeneous half-space case due to the effect of finite thickness. Thus, it should be noted that, in practice, the solution of a half-space case cannot be used to represent the one of a finite body case.

The effect of thickness of the graded layer on the distribution of contact stresses is plotted in Fig. $11(a-c)$ for the case of a flat punch, where one can see that the normal contact stress $p(x) / \sigma_{0}$ distributes more uniformly in cases of $\mu_{1} / \mu_{3}=1 / 8$ and $\mu_{1} / \mu_{3}=1$ when the thickness decreases, while it is contrary in the case of $\mu_{1} / \mu_{3}=8$. The corresponding stress intensity factors $F_{l}(b) / F_{0}$ decrease with a decreasing thickness $h / b$ in cases of $\mu_{1} / \mu_{3}<1$ and $\mu_{1} /$ $\mu_{3}=1$, but increase in the case of $\mu_{1} / \mu_{3}>1$ as shown in Table 1 . The results also demonstrate that the contact stress in a finite model cannot be accurately represented by that in a half-space one. However, if the thickness of the finite layer is much large, the boundary effect is weakened whether in cases of $\mu_{1} / \mu_{3}<1$ and $\mu_{1} / \mu_{3}=1$ or in the case of $\mu_{1} / \mu_{3}>1$. Thus, it is reasonable to use a layer with large enough thickness to represent a half-space in the numerical simulations.

Distributions of the non-dimensional normal and tangential contact stresses, $p(x) / \mu_{1}$ and $q(x) / \mu_{1}$, under a circular punch are shown in Fig. 12(a-f) for different types of $\mu_{1} / \mu_{3}$. From Fig. 12(a-f), one can see that, for a fixed contact width, the normal contact stress increases with a decreasing layer thickness, which is totally different from that in the flat punch case. It can be inferred that, for a determined external load, the contact width will decrease with a decreasing layer thickness in such as circular punch case. This phenomenon agrees qualitatively with that in the model of a homogeneous layer (Nowell and Hills, 1988).

In the present paper, we only focus on the contact stress and stick zone induced by an externally normal load. In fact, the externally tangential load in any fretting problem is inevitably involved, the results of which can also be found by a similar method of the present paper.

\section{Conclusions}

A more general and practical model has been established in the present paper for the slip contact problem between a rigid stamp and a finite graded layer. Exponential variation law in the thickness direction is adopted for the layer's shear modulus. The governing equation establishing the relation between the contact stress and the contact 
(a)
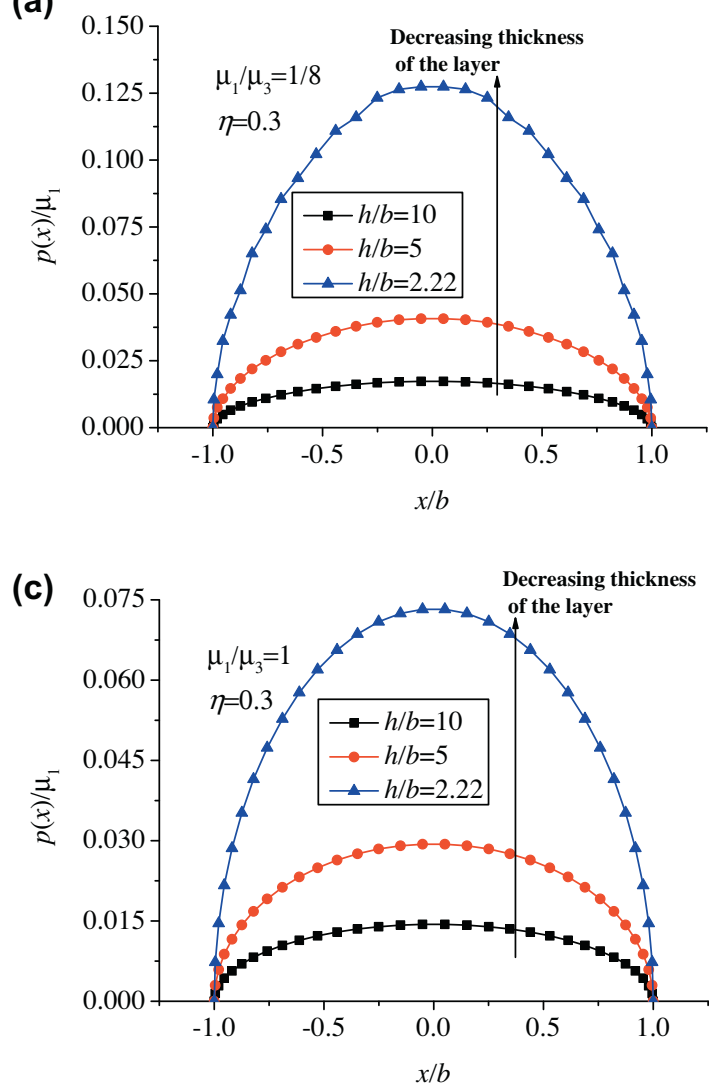

(e)

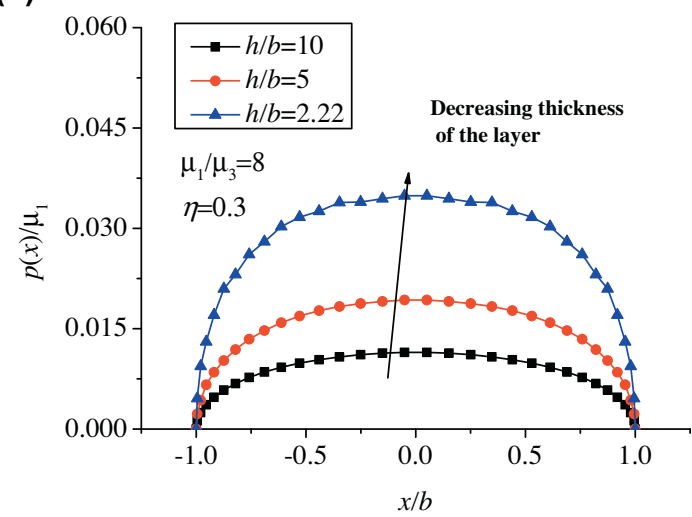

(b)

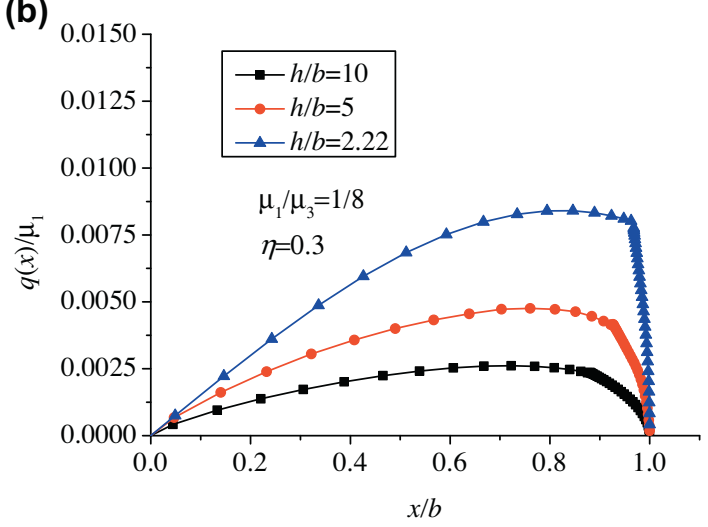

(d)

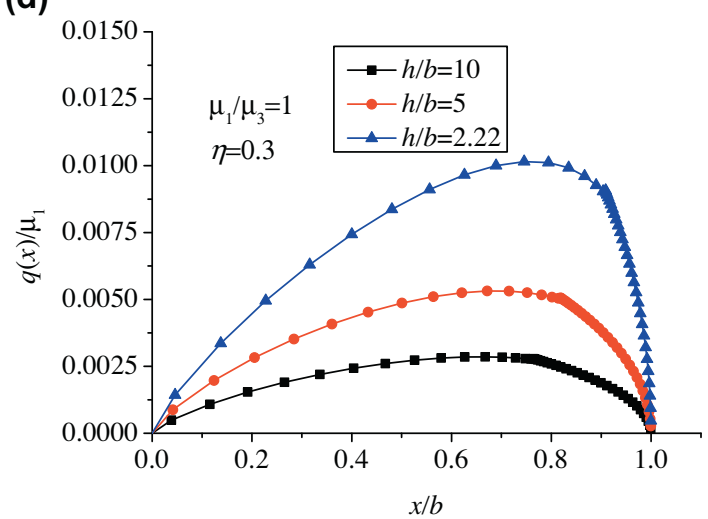

(f)

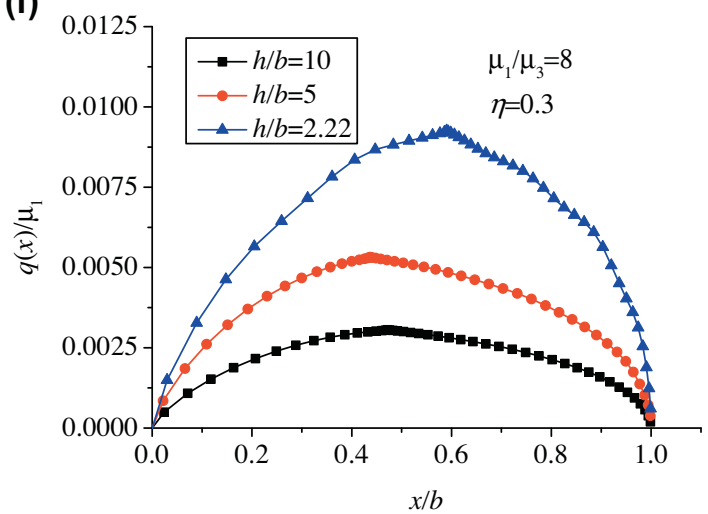

Fig. 12. Distributions of normalized contact stress distribution in the case of a circular punch for some selected thickness of the graded layer with $\eta=0.3$ and $v=0.3$ (a) normal stress $\left(\mu_{1} / \mu_{3}=1 / 8\right)$; (b) shear stress $\left(\mu_{1} / \mu_{3}=1 / 8\right)$; (c) normal stress $\left(\mu_{1} / \mu_{3}=1\right)$; (d) shear stress $\left(\mu_{1} / \mu_{3}=1\right)$; (e) normal stress $\left(\mu_{1} /\right.$ $\left.\mu_{3}=8\right)$; (f) shear stress $\left(\mu_{1} / \mu_{3}=8\right)$.

width is derived for the slip contact models with arbitrary punch-tips. Two special cases, i.e., a flat punch case and a circular punch one, are analyzed in details. It was found that the distributions of the contact stresses and the size of the stick zone are significantly influenced by the shear modulus of the graded layer and the friction coefficient of the contact interface. Furthermore, it is interesting to find that the finite thickness of the graded layer leads to significant influence on the contact stresses and the scale of stick zone too, which demonstrates that the solution of the slip contact model of a half-space cannot be valid for the contact model of a finite layer. With the increasing thickness of the layer, the solution of the present finite model tends to the one of the half-space model. The results 
in this paper should be helpful in the design of new graded materials for fretting fatigue and useful in applications of practical engineering.

\section{Acknowledgment}

This work is supported by the National Science Foundation of China (Nos. 10972220, 11125211 and 11021262) and the Project No. 2012CB937500.

\section{References}

Araújo, J.A., Nowell, D., 1999. Analysis of pad size effects in fretting fatigue using short crack arrest methodologies. Int. J. Fatigue 21, 947-956.

Argatov, I.I., 2001. The pressure of a punch in the form of an elliptic paraboloid on an elastic layer of finite thickness. Journal of Applied Mathematics and Mechanics 65, 495-508.

Bramhall, R., 1973. Studies in Fretting Fatigue. University of Oxford, UK.

Chen, P., Chen, S., 2012. Contact behaviors of a rigid punch and a homogeneous half-space coated with a graded layer. Acta Mech. 223, 563-577.

Chen, S.H., Yan, C., Soh, A., 2009a. Adhesive behavior of two-dimensional power-law graded materials. Int. J. Solids Struct. 46, 3398-3404.

Chen, S.H., Yan, C., Zhang, P., Gao, H.J., 2009b. Mechanics of adhesive contact on a power-law graded elastic half-space. J. Mech. Phys. Solids 57, 1437-1448.

Choi, H.J., Paulino, G.H., 2008. Thermoelastic contact mechanics for a flat punch sliding over a graded coating/substrate system with frictional heat generation. J. Mech. Phys. Solids 56, 1673-1692.

Choi, H.J., Paulino, G.H., 2010. Interfacial cracking in a graded coating/ substrate system loaded by a frictional sliding flat punch. Proceedings of the Royal Society A - Mathematical, Physical and Engineering Sciences 466, 853-880.

Ciavarella, M., 1998. The generalized Cattaneo partial slip plane contact problem. I - theory. Int. J. Solids Struct. 35, 2349-2362.

Ciavarella, M., Hills, D., 1999. Brief note: some observations on oscillating tangential forces and wear in general plane contacts. European Journal Mechanics A/Solids 18, 491-497.

Dini, D., Nowell, D., 2004. Flat and rounded fretting contact problems incorporating elastic layers. Int. J. Mech. Sci. 46, 1635-1657.

El-Borgi, S., Abdelmoula, R., Keer, L., 2006. A receding contact plane problem between a functionally graded layer and a homogeneous substrate. Int. J. Solids Struct. 43, 658-674.

Elloumi, R., Kallel-Kamoun, I., El-Borgi, S., 2010. A fully coupled partial slip contact problem in a graded half-plane. Mech. Mater. 42, 417-428.

Erdogan, F., Gupta, G.D., Cook, T.S., 1973. Numerical solution of singular integral equations. In: Sih, G.C. (Ed.), Mechanics of Fracture, vol. 7. Noordhoff, Leyden, pp. 368-425.

Fellows, L.J., Nowell, D., Hills, D.A., 1995. Contact stresses in a moderately thin strip (with particular reference to fretting experiments). Wear $185,235-238$

Giannakopoulos, A.E., Pallot, P., 2000. Two-dimensional contact analysis of elastic graded materials. J. Mech. Phys. Solids 48, 1597-1631.

Giannakopoulos, A.E., Suresh, S., 1997a. Indentation of solids with gradients in elastic properties. 1. Point force. Int. J. Solids Struct. 34, 2357-2392.
Giannakopoulos, A.E., Suresh, S., 1997b. Indentation of solids with gradients in elastic properties. 2. Axisymmetric indentors. Int. J. Solids Struct. 34, 2393-2428.

Guler, M.A., Erdogan, F., 2004. Contact mechanics of graded coatings. Int. J. Solids Struct. 41, 3865-3889.

Guler, M.A., Erdogan, F., 2006. Contact mechanics of two deformable elastic solids with graded coatings. Mech. Mater. 38, 633-647.

Guler, M.A., Erdogan, F., 2007. The frictional sliding contact problems of rigid parabolic and cylindrical stamps on graded coatings. Int. J. Mech. Sci. 49, 161-182.

Guo, X., Jin, F., Gao, H.J., 2011. Mechanics of non-slipping adhesive contact on a power-law graded elastic half-space. Int. J. Solids Struct. 48, 2565-2575.

Hanson, M., Keer, L., 1989. Cyclic tangential loading of dissimilar elastic bodies. Int. J. Mech. Sci. 31, 107-119.

Jin, F., Guo, X., 2010. Non-slipping adhesive contact of a rigid cylinder on an elastic power-law graded half-space. Int. J. Solids Struct. 47, 15081521.

Jitcharoen, J., Padture, N.P., Giannakopoulos, A.E., Suresh, S., 1998. Hertzian-crack suppression in ceramics with elastic-modulusgraded surfaces. J. Am. Ceram. Soc. 81, 2301-2308.

Johnson, K.L., 1985. Contact Mechanics. Cambridge University Press.

Kallel-Kamoun, I., Elloumi, R., El-Borgi, S., 2010. Partial slip contact problem in a graded half Plane. J. Comput. Theor. Nanosci. 7, 367-378.

Ke, L.L., Wang, Y.S., 2010. Fretting contact of two dissimilar elastic bodies with functionally graded coatings. Mech. Adv. Mater. Struct. 17, 433447.

Krumova, M., Klingshirn, C., Haupert, F., Friedrich, K., 2001. Microhardness studies on functionally graded polymer composites. Compos. Sci. Technol. 61, 557-563.

Liu, J., Ke, L.L., Wang, Y.S., 2011. Two-dimensional thermoelastic contact problem of functionally graded materials involving frictional heating. Int. J. Solids Struct. 48, 2536-2548.

Muskhelishvili, N.L., 1953. Singular Integral Equations. P. Noordhoff, Groningen.

Nowell, D., 1988. An Analysis of Fretting Fatigue. University of Oxford, UK.

Nowell, D., Hills, D., Sackfield, A., 1988. Contact of dissimilar elastic cylinders under normal and tangential loading. J. Mech. Phys. Solids 36, 59-75.

Nowell, D., Hills, D.A., 1988. Contact problems incorporating elastic layers. Int. J. Solids Struct. 24, 105-115.

Rhimi, M., El-Borgi, S., Ben Said, W., Ben Jemaa, F., 2009. A receding contact axisymmetric problem between a functionally graded layer and a homogeneous substrate. Int. J. Solids Struct. 46, 3633-3642.

Spence, D., 1973. An Eigenvalue Problem for Elastic Contact with Finite Friction. Cambridge University Press, pp. 249-268.

Spence, D.A., 1968. Self similar solutions to adhesive contact problems with incremental loading. Proc. R. Soc. A 305, 55-80.

Suresh, S., Giannakopoulos, A.E., Alcala, J., 1997. Spherical indentation of compositionally graded materials: theory and experiments. Acta Mater. 45, 1307-1321.

Suresh, S., Mortensen, A., 1998. Fundamentals of functionally graded materials: processing and thermomechanical behaviour of graded metals and metal-ceramic composites. Book - Inst. Mater., 698.

Wang, Z.J., Wang, W.Z., Wang, H., Zhu, D., Hu, Y.Z., 2010. Partial slip contact analysis on three-dimensional elastic layered half space. J. Tribol. 132, 021403.

Xing, A., Jun, Z., Chuanzhen, H., Jianhua, Z., 1998. Development of an advanced ceramic tool material - functionally gradient cutting ceramics. Mater. Sci. Eng., A 248, 125-131. 